\title{
Anterior chamber angle tears after non-perforating injury
}

\author{
DAVID MOONEY \\ Croydon Eye Unit, Croydon, Surrey
}

Recession of the anterior chamber angle is a common finding after ocular contusion and a small percentage of these injured eyes eventually develop secondary glaucoma, frequently more than Io years after the injury. It is desirable to detect this small group of glaucoma cases at an early stage so that appropriate therapy can be instituted before vision has been seriously impaired.

The purpose of this paper is to report the incidence and extent of angle tears and to attempt to determine which cases require long-term follow-up.

Angle recession was first reported by Collins (1892). He described the pathological findings in two eyes enucleated after non-perforating injuries; his paper included microphotographs showing a split between the circular and longitudinal muscle fibres of the ciliary body with widening of the angle and deepening of the anterior chamber.

D'Ombrain (1945, 1946, I949) drew attention to trauma as a cause of unilateral chronic glaucoma but did not describe the gonioscopic or pathological findings.

Wolff and Zimmerman ( 1962 ) restored interest in the subject by reporting "moderate to marked" angle recession in 6 per cent. of a series of 300 consecutive enucleations with a history of previous contusion. Their pathological findings in early cases included lacerations into the face of the ciliary muscle with separation of the circular from the longitudinal fibres, disruption of the trabecular meshwork, and in long-standing cases, atrophy of the circular muscle fibres with retrodisplacement of the iris root and pars plicata, and advanced degeneration, atrophy, and replacement fibrosis of the trabecular meshwork; in many cases there was a newly-formed hyaline membrane covering the inner surface of the trabeculae, similar to that described by Reese (1944).

Jensen ( 1968 ) reported angle recession in 4.8 per cent. of enucleated traumatized eyes and again described atrophy and fibrosis of the ciliary muscle and trabecular meshwork.

Several authors have reported the incidence of angle recession in traumatic hyphaema (Table I).

\section{Table I Angle recession after traumatic hyphaema}

\begin{tabular}{|c|c|c|c|c|}
\hline \multirow{2}{*}{ Author } & \multirow{2}{*}{ Date } & \multirow{2}{*}{$\begin{array}{l}\text { No. of } \\
\text { hyphaema } \\
\text { cases }\end{array}$} & \multicolumn{2}{|c|}{$\begin{array}{l}\text { Cases with angle } \\
\text { recession }\end{array}$} \\
\hline & & & No. & Per cent. \\
\hline Blanton & I964 & 182 & 130 & 71 \\
\hline Howard and others & 1965 & 50 & 47 & 94 \\
\hline Britten & 1965 & 54 & 10 & 20 \\
\hline Tönjum & 1966 & 53 & 50 & 94 \\
\hline Thorkilgaard and Moestrup & 1967 & 44 & 20 & 45 \\
\hline Spaeth & 1967 & 48 & 30 & 61 \\
\hline Tönjum & 1968 & 160 & 160 & 100 \\
\hline
\end{tabular}

Table II Age of 53 patients with hyphaema

\begin{tabular}{|c|c|}
\hline Age (yrs) & No. of cases \\
\hline Under 10 & 7 \\
\hline $10-19$ & 22 \\
\hline $20-29$ & 12 \\
\hline $30-39$ & 2 \\
\hline $40-49$ & 5 \\
\hline 50 and over & 5 \\
\hline Total & 53 \\
\hline
\end{tabular}


Uniocular open-angle glaucoma has been described in cases of angle recession by several authors:

Wolff and Zimmerman (1962) reported three cases of postcontusion glaucoma diagnosed many years after the injury: all had angle recession.

Pettit and Keates (1963) described eight cases of blunt ocular injury, in one of which uniocular glaucoma developed 8 years after the trauma.

Alper (1963) reviewed a selected series of 27 cases of angle recession and fourteen of these had uniocular glaucoma. Eight were diagnosed within 4 years of the injury and the remaining six more than 14 years after the original contusion. Thirteen had angle recession extending around $240^{\circ}$ or more of the circumference of the angle; the remaining case had $150^{\circ}$ of recession.

Blanton ( 1964 ) found nine patients with uniocular glaucoma in 130 cases of angle recession (an incidence of 7 per cent.). Five were diagnosed within 3 years of injury, but the remaining four more than Io years after the injury. He found no correlation between the glaucoma and the degree of angle recession in the early group, but all four late cases of glaucoma had more than $180^{\circ}$ of recession. He stressed the point that there appeared to be two peak incidences of glaucoma in angle recession cases, one within a year or so of injury and the other to or more years after the injury. The findings of Alper (1963) would seem to support this.

Howard, Hutchinson, and Frederick (1965) performed tonography and applanation tonometry on 25 of their fifty hyphaema cases within 4 months of injury and found four patients with ocular tensions of $30 \mathrm{~mm} . \mathrm{Hg}$ or more, all with moderate to deep tears extending around the entire circumference of the angle.

Tönjum ( 1968 ), in a study of 160 cases of hyphaema with angle recession, described late changes in tension and facility of outflow occurring $\mathrm{I}$ to 16 years after injury. He found a significant correlation between the extent of the angle recession and the decreased outflow facility of the injured eyes compared with the normal fellow eyes. He also found two cases of uniocular glaucoma, one diagnosed a few weeks after injury but the other diagnosed 6 years after injury, and both had angle recession of more than $270^{\circ}$.

\section{Material and methods}

Sixty cases of hyphaema due to non-perforating injury were admitted to the Croydon Eye Unit between May, 1968, and May, 1971, and 53 of them (10 women and 43 men) attended for examination. The age incidence is shown in Table II.

The interval between the date of injury and the date of examination varied from 2 weeks to 3 years.

Examination consisted of applanation tonometry and gonioscopy. Both angles were examined at the same sitting, corresponding areas of the injured and normal angles were compared, and goniophotographs were taken of selected cases.

Howard and others (1965) classified tears in the angle of the anterior chamber as shallow, moderate, and deep; they recorded the circumferential extent of these tears in degrees:

\section{(1) Shallow tears}

"Consisted of separation of the processes of the uveal meshwork so that the ciliary body band and the scleral spur were 'bared', that is, more plainly visible than in the fellow eye. The separation of these processes leaves pigmented tags on the anterior surface of the peripheral iris, on the ciliary body band, on the scleral spur, and on the posterior portion of the trabecular meshwork ... The ciliary body band appears darker and wider and the scleral spur appears whiter than the same structure in the fellow eye... In shallow tears, no actual traumatic cleft into the face of the ciliary body is present."

\section{(2) Moderate tears}

"Characterized by a definite cleft into the fibres of the ciliary muscle ... Whenever such a moderate tear is present the angle appears deeper than the angle of the other eye..." 


\section{(3) Deep tears}

"Characterized by a fissure in the ciliary body when the apex of the fissure cannot be seen gonioscopically."

Shallow tears were visible at either extremity of the moderate and deep tears.

In the present study Howard's classification has been followed, though "Shallow Tears" are described as "Grade I Tears", "Moderate Tears" as "Grade II Tears", and "Deep Tears" as "Grade III Tears". The circumferential extent of the tears has been recorded in degrees.

Anatomically, the trabecular meshwork consists of two parts: the outer corneo-scleral trabeculae, which run from Schwalbe's line to the scleral spur, and the inner uveal meshwork, which runs from Schwalbe's line across the scleral spur to terminate in the anterior surface of the ciliary body and the root of the iris. In a few of the cases recorded in the present series it was difficult to differentiate between tears of the uveal meshwork overlying the ciliary muscle (i.e. Grade I Tear) and shallow tears into the ciliary muscle itself (i.e. Grade II Tear); however when doubt existed the tear was recorded as Grade $I$ in these circumstances.

\section{Results}

Of the 53 patients examined, eleven (2 I per cent.) had normal angles, 4 I (77 per cent.) had angle tears, and one had extensive peripheral anterior synechiae.

The $4^{\text {I }}$ cases with angle tears were assessed with regard to the grade; fourteen patients had Grade I tears only, nineteen Grade II, and eight a combination of Grades II and III. $\vec{\varphi}$ Many cases with Grade II and Grade II + III tears also had Grade I tears at their N extremities but these minor tears were not recorded.

The circumferential extent of the tears is shown in Tables III and IV.

Table III Circumferential extent of Grade I and Grade II tears in 33 cases

\begin{tabular}{|c|c|c|c|c|c|}
\hline Grade & $<90^{\circ}$ & $<180^{\circ}$ & $<270^{\circ}$ & $270^{\circ} \rightarrow 360^{\circ}$ & Total \\
\hline I & II & 3 & o & o & 14 \\
\hline II & 5 & 6 & 4 & 4 & 19 \\
\hline Total & 16 & 9 & 4 & 4 & 33 \\
\hline
\end{tabular}

Table IV Circumferential extent of eight Grade II and Grade III tears

\begin{tabular}{|c|c|c|c|}
\hline Case no. & Grade III & Grade II & Total extent of tear $\left({ }^{\circ}\right)$ \\
\hline $\mathbf{I}$ & $60^{\circ}$ & $210^{\circ}$ & $270^{\circ}$ \\
\hline 2 & $270^{\circ}$ & $90^{\circ}$ & $360^{\circ}$ \\
\hline 3 & $15^{\circ}$ & $120^{\circ}$ & $135^{\circ}$ \\
\hline 4 & $60^{\circ}$ & $300^{\circ}$ & $360^{\circ}$ \\
\hline 5 & $90^{\circ}$ & $30^{\circ}$ & $120^{\circ}$ \\
\hline 6 & $90^{\circ}$ & $75^{\circ}$ & $165^{\circ}$ \\
\hline 7 & $30^{\circ}$ & $195^{\circ}$ & $225^{\circ}$ \\
\hline 8 & $75^{\circ}$ & $60^{\circ}$ & $135^{\circ}$ \\
\hline
\end{tabular}


Fig. I A shows normal angle structures on the left side of the diagram merging into an area with Grade I angle tears on the right with ruptured iris processes $(a)$ and a tear into

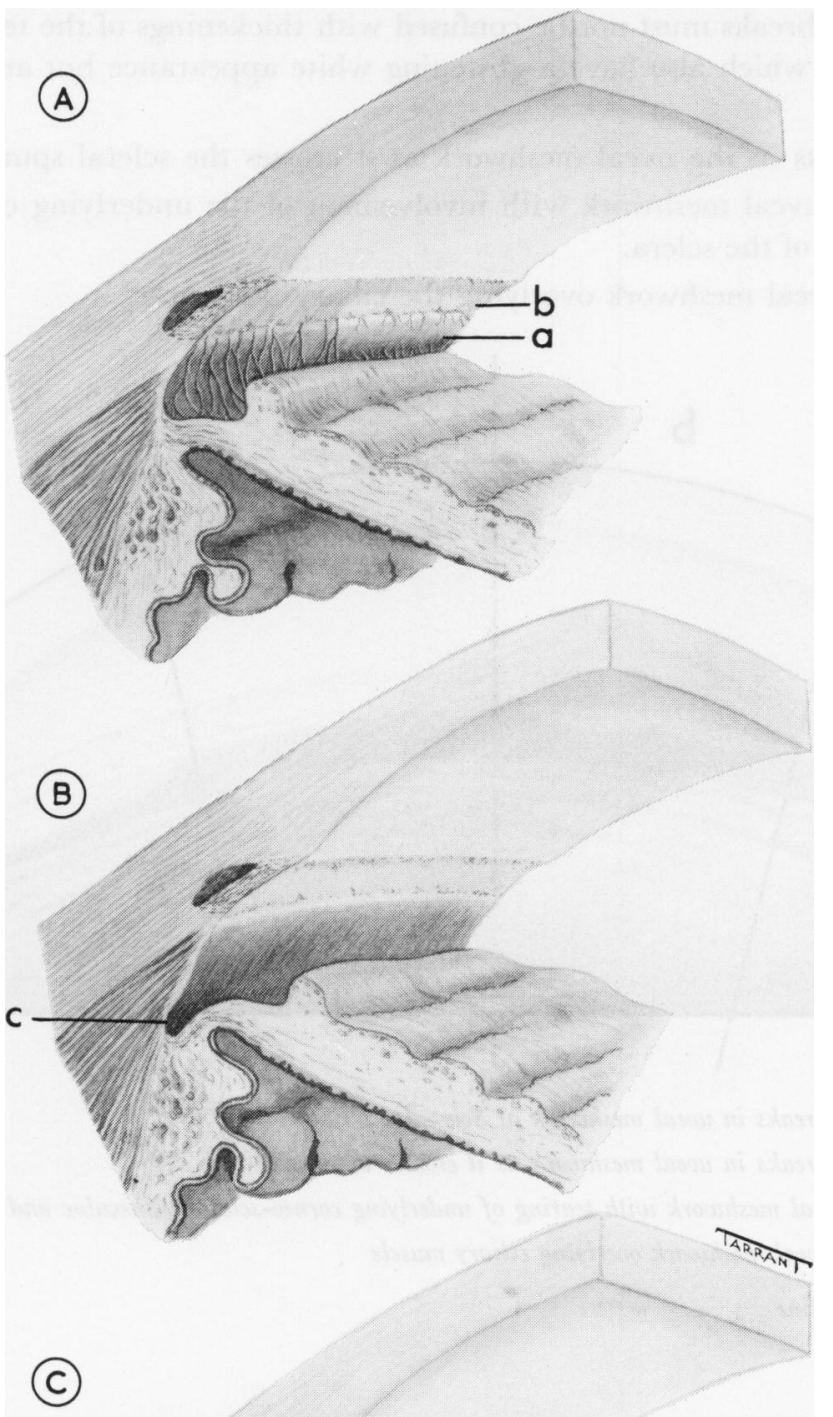
the uveal meshwork overlying the corneo-scleral trabeculae (b). Fig. I B shows a Grade II angle tear with widening of the angle and a split into the ciliary muscle $(c)$. Fig. IC shows a Grade III angle tear with marked widening of the ciliary band and a deep split into the ciliary muscle, the apex of which is out of gonioscopic view $(d)$.

FIG. I (A) Normal angle on left side. Grade I tears, consisting of torn iris processes at " $a$ " and torn uveal meshwork at " $b$ ", on right side of drawing

(B) Grade II angle tear with tear into ciliary muscle at " $c$ " and widening of ciliary body band

(C) Grade III tear with a deep tear into ciliary muscle at " $d$ " and marked widening of angle

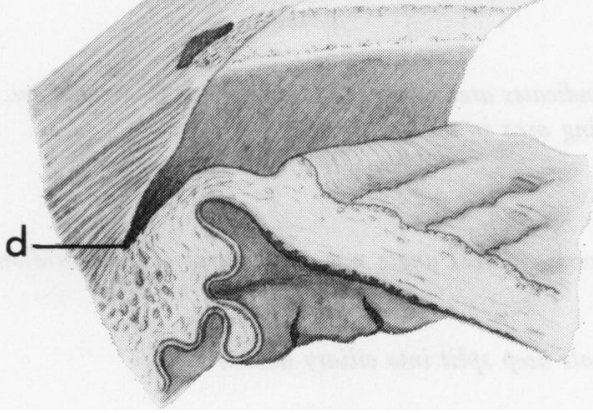


Fig. 2 is a composite drawing of some of the Grade I tears seen in this series:

a: Semilunar breaks in the uveal meshwork at Schwalbe's line were seen in a few cases; these white semilunar breaks must not be confused with thickenings of the termination of Descemet's membrane which also have a glistening white appearance but are commonly seen in normal eyes.

$b$ : Semilunar breaks in the uveal meshwork as it crosses the scleral spur.

$c$ : A break in the uveal meshwork with involvement of the underlying corneo-scleral trabeculae and baring of the sclera.

$d$ : Breaks in the uveal meshwork overlying the ciliary body band.

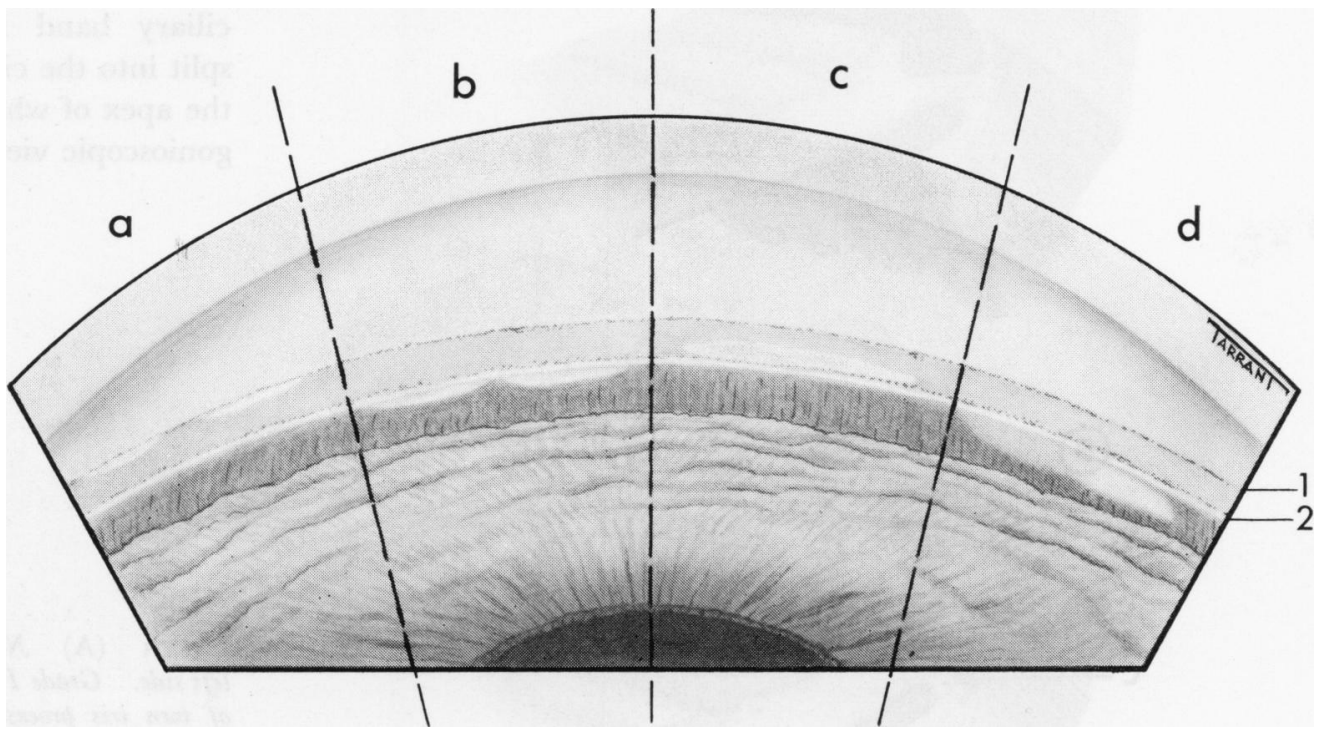

FIG. 2 (a) Semilunar breaks in uveal meshwork at Schwalbe's line

(b) Semilunar breaks in uveal meshwork as it crosses the scleral spur

(c) Break in uveal meshwork with tearing of underlying corneo-scleral trabeculae and baring of sclera

(d) Breaks in uveal meshwork overlying ciliary muscle

(1) Schwalbe's line

(2) Scleral spur

Figs 3 to 8 (opposite) are goniophotographs of cases with angle tears.

FIG. 3 Normal angle

FIG. 4 (Same patient as Fig. 3). Arrow indicates area of Grade II angle tear in injured eye. Note widening of ciliary body band compared with corresponding area in the normal eye

FIG. 5 Normal angle

FI G. 6 (Same patient as Fig. 5). Extensive Grade III angle tear. There is also a meridional tear in the iris stroma (arrowed)

FIG. 7 Grade II angle tear. Arrows indicate deep split into ciliary muscle

FIG. 8 Grade II angle tear. Arrow indicates split in ciliary muscle 
(3)

(4)
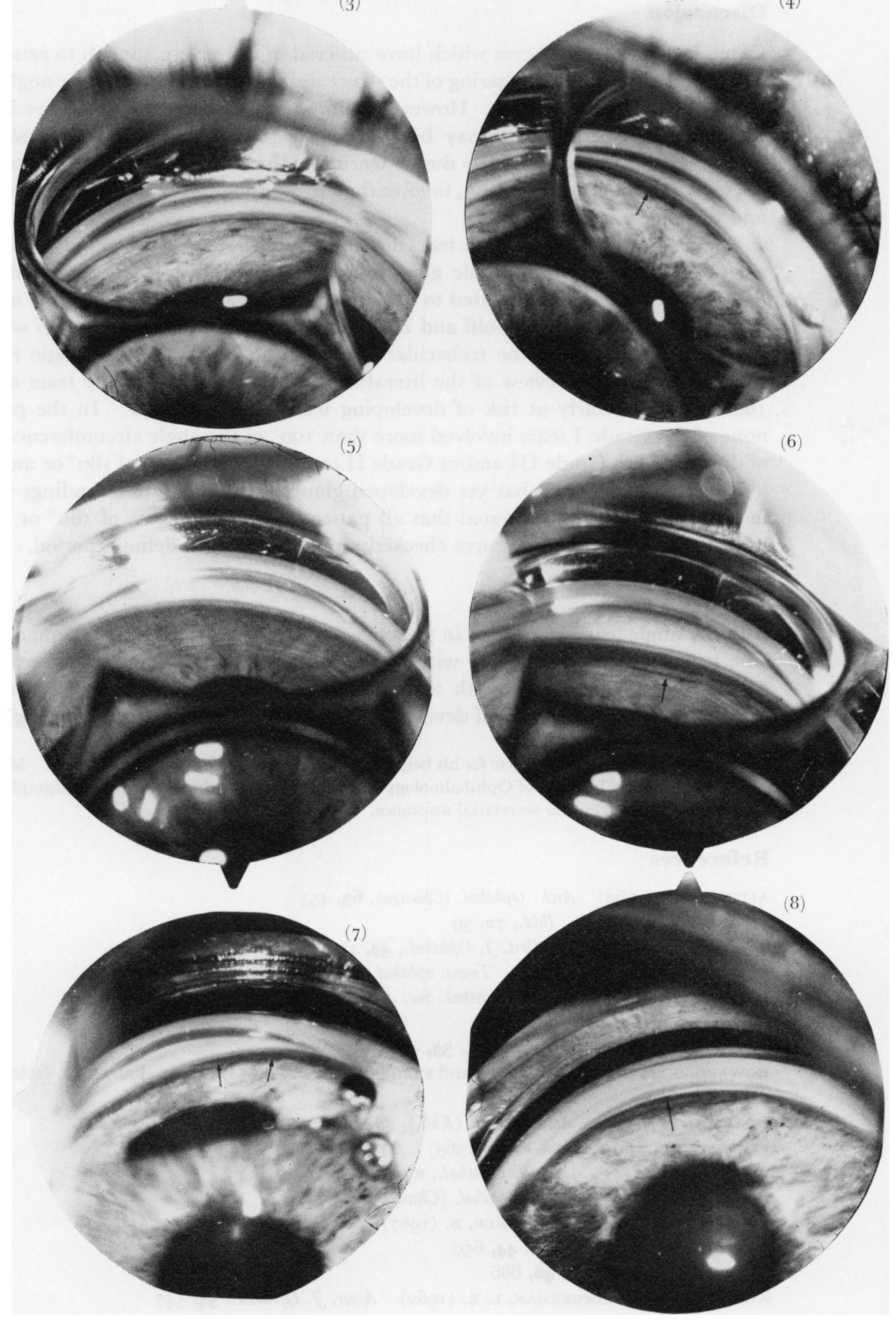

$\underline{\underline{w}}$

c

응

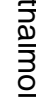

寻

.

政 


\section{Discussion}

Careful examination of eyes which have suffered injury severe enough to cause hyphaema will reveal some degree of tearing of the structures of the anterior chamber angle in approximately 80 per cent. of cases. However, comparison with the uninjured eye is essential or the milder grades of tears may be overlooked. As described by Howard and others (1965), hyphaema is frequently due to tearing of the blood vessels in the anterior chamber angle and this must, of necessity, involve the surrounding tissues such as the uveal meshwork and the ciliary muscle.

The majority of cases of angle tears develop no serious long-term complications: however, a few cases do develop chronic glaucoma in the injured eye and the likelihood of this development seems to be related to the severity of the angle damage; this is not surprising in view of the findings of Wolff and Zimmerman (1962) and Jensen (1968) who described atrophy and fibrosis of the trabecular meshwork in cases of severe angle recession. It would seem from a review of the literature that patients with angle tears of more than $180^{\circ}$ are particularly at risk of developing secondary glaucoma. In the present series, none of the Grade I tears involved more than $100^{\circ}$ of the angle circumference, but twelve of the cases with Grade III and/or Grade II tears had angle tears of $180^{\circ}$ or more.

No case in this series has yet developed glaucoma (applanation readings were normal in all cases), but it is suggested that all patients with angle tears of $180^{\circ}$ or more should have their intraocular pressures checked annually for an indefinite period.

\section{Summary}

Anterior angle tears were found in 77 per cent. of a series of 53 cases of traumatic hyphaema. The tears have been classified with regard to depth and circumferential extent and it is suggested that all patients with tears of $180^{\circ}$ or more should be followed up annually, as this group is most at risk of developing post-traumatic uniocular open-angle glaucoma.

I wish to thank Mr. Dermot Pierse for his help and advice in the preparation of this paper. Mr. Tarrant and Mr. Fletcher of the Institute of Ophthalmology kindly prepared the drawings and photographs. My thanks are due to Mrs. S. Tant for secretarial assistance.

\section{References}

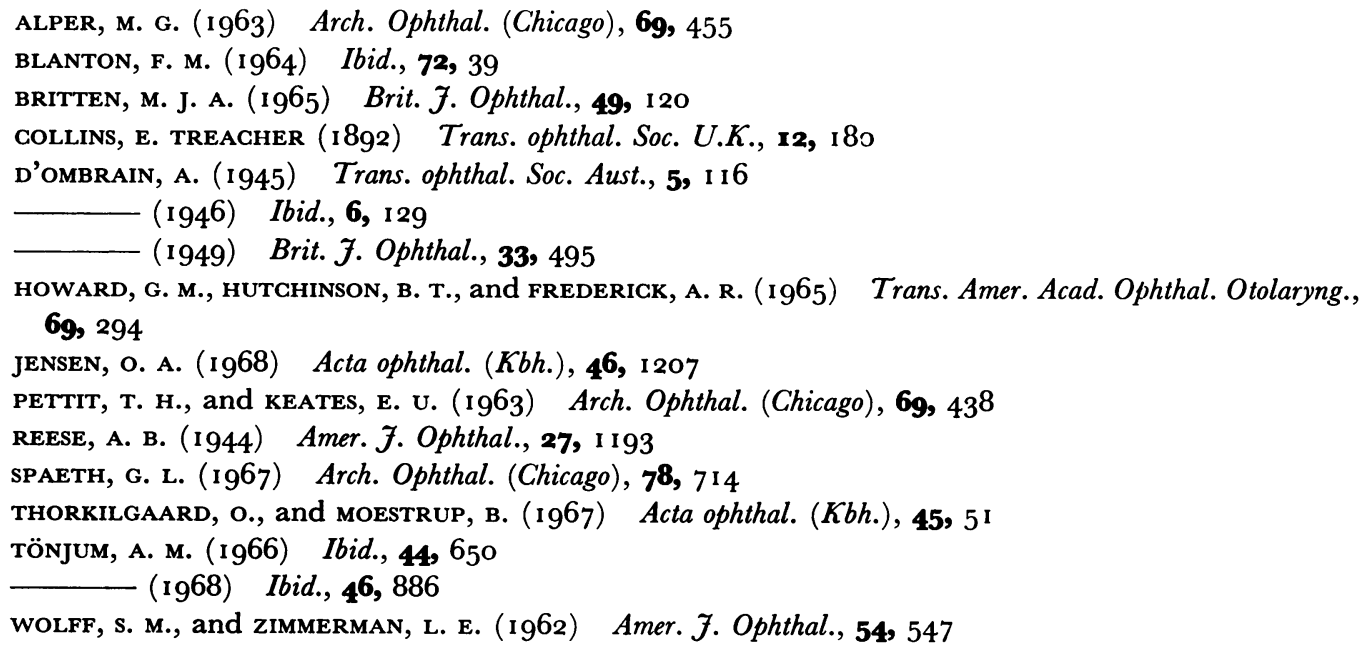

